INSECTOS PERJUDICIALES Y BENÉFICOS EN CULTIVO DE CÁRTAMO EN LA REGIÓN PAMPEANA SEMIÁRIDA

\title{
HARMFUL AND BENEFICIAL INSECTS FOR SAFFLOWER CROP IN THE SEMIARID PAMPEAN REGION
}

\author{
Fritz Florencia ${ }^{1}$, Fernando Ramonda ${ }^{1}$ \\ \& Estela M. Baudino ${ }^{1^{*}}$
}

Recibido 28/03/2018

Aceptado 12/09/2018

\begin{abstract}
RESUMEN
Los objetivos del trabajo fueron identificar las especies de insectos perjudiciales y benéficas y estudiar su distribución temporal en relación al estado fenológico del cultivo de cártamo. Las especies identificadas como perjudiciales fueron: Uroleucon jaceae (L)., Capitophorus elaeagni (del Guercio), Frankliniella occidentalis P., Dichelops furcatus (F.), Athaumasthus haematicus (Stål), Nezara viridula (L.), Nysius simulans Stål, Edessa meditabunda (F.), Piezodorus guildinii (W.), Helicoverpa zea B., Epicauta adspersa K., Spodoptera frugiperda (J. E. Smith), Chauliognathus scriptus (Germ.), Pantomorus auripes H. y Rachiplusia nu (G.). Con respecto a los pulgones, la mayor densidad se observó en el estado de ramificación, ocurrido a principios de noviembre, atacando preferentemente el tercio superior de la planta: hojas, brotes tiernos y capítulos. La chinche roja, Athaumasthus haematicus (Stål), representó la mayor proporción del total de chinches perjudiciales encontradas y fue detectada antes de los pulgones, desde principio de elongación del tallo. En cuanto a las especies benéficas se identificaron seis especies de insectos: Hippodamia convergens G., Eriopis connexa G., Harmonia axyridis (P.), Nabis sp., Ophion sp. y Apis melífera L., además de varias especies de arañas. Los coccinélidos Hippodamia convergens, Eriopis connexa y Harmonia axyridis fueron los principales agentes de control natural.
\end{abstract}

PALABRAS CLAVE: Carthamus tinctorius L, pulgones, chinches, coccinélidos, estado fenológico, enemigos naturales

\begin{abstract}
The work's aims were to identify both harmful and helpful insect species for safflower crop and to study their distribution along time in relation to plant phenological state. The insect species identified as harmful were: Uroleucon jaceae L., Capitophorus elaeagni, Frankliniella occidentalis P., Dichelops furcatus (F.), Athaumasthus haematicus (Stål), Nezara viridula (L.), Nysius simulans Stål, Edessa meditabunda (F.), Piezodorus guildinii (W.), Helicoverpa zea B., Epicauta adspersa K., Spodoptera frugiperda (J. E. Smith), Chauliognathus scriptus (Germ.), Pantomorus auripes H. and Rachiplusia nu (G.). Regarding aphids, their highest density was observed at the beginning of November, when crop was in the state of branching, with invasion mainly of the upper third of plants: leaves, young shoots and flower heads. Among all harmful true bugs found in the crop, red bug Athaumastus haematicus (Stål)- comprised the highest proportion and was detected before aphids attacks, when stem elongation of plants was just beginning. As for beneficial species, the six insects detected in sufflower crop were Hippodamia convergens G., Eriopis connexa G., Harmonia axyridis (P.), Nabis sp., Ophion sp. and Apis melifera L., besides various spider species. The coccinellidsHippodamia convergens; Eriopis connexa and Harmonia axydiris performed as the main natural control agents at crop.
\end{abstract}

KEY WORDS: Carthamus tinctorius L, aphids, true bugs, coccinellids, phenological state, natural enemies

\section{Cómo citar este trabajo:}

Fritz F., F. Ramonda \& E.M. Baudino. 2018. Insectos perjudiciales y benéficos en cultivo de cártamo en la región pampeana semiárida. Semiárida 28(2): 35-44.

\section{INTRODUCCIÓN}

El cártamo (Carthamus tinctorius L.), también conocido como alazor o falso azafrán es una planta anual, de la familia Asteraceae. Es pa-

1 Universidad Nacional de La Pampa, Facultad de Agronomía, La Pampa

* baudino@agro.unlpam.edu.ar 
recido a los cardos y está adaptado a climas cálidos y secos, como los de la región semiárida pampeana. Es originario del Mediterráneo oriental y centro de Asia central, que comprende el Noroeste de la India (Punjab), Afganistán, Cachemira, Tadzhikistán y Kirguistán. Originalmente se lo cultivaba como planta tintórea, usando los pigmentos de las flores en la industria textil y alimenticia (Carpintero \& Dughetti, 2012).

Las cabezuelas florales o capítulos, se encuentran en la parte terminal de tallos ramificados; su color es rojo, naranja, amarillo o blanco. Cada capítulo puede tener entre 15 a 30 semillas. Éstas son similares a las del girasol pero algo más cortas, su color normalmente es blanco pero también puede ser grisáceo (Rivas \& Matarazzo, 2009).

Actualmente ha tomado relevancia por ser considerado uno de los cultivos oleaginosos anuales más resistente a sequía. La importancia de este cultivo radica en las propiedades de su aceite. Hay dos tipos de cártamo que producen diferentes tipos de aceite: uno alto en ácido graso monoinsaturado (ácido oleico) y otro alto en ácido graso poliinsaturado (ácido linoleico).

El aceite de los cultivares de cártamo alto oleico se utiliza para la alimentación, ya que es similar al aceite de girasol, muy usado como aceite de cocinar, en ensaladas, para producir margarinas y también como suplemento nutricional. Las flores de cártamo se usan ocasionalmente en la gastronomía como un sustituto barato del azafrán. También sus semillas se usan comúnmente como alternativa a las del girasol en la alimentación de aves y mamíferos. El aceite de cártamo con mayor contenido de ácido linoleico se usa en pinturas, en lugar del aceite de linaza particularmente con pintura blanca, ya que no trasmite el tinte amarillo que posee la linaza (Emongor et al., 2010).

Los principales países donde se cultiva el cártamo son la India, EE.UU., México y, en menor medida, Etiopía, Argentina, China y Australia (Carpintero \& Dughetti, 2012). En la Argentina se lo cultiva en las provincias de Salta, Santiago del Estero y Chaco; hace unos años se lo hacía en Catamarca, Jujuy y Tucumán (Rivas \& Matarazzo, 2009).

Este cultivo fue promovido en el país por el
Ing. Agr. Guillermo Covas, del INTA de Anguil, La Pampa, en la década del `60. Existen antecedentes de buen comportamiento en la región semiárida pampeana. Sin embargo, no se consolidó en la región; el motivo principal fue el desarrollo del cultivo de girasol, con un mercado mayor y firme que terminó por ocupar ese espacio (Rivas \& Matarazzo, 2009). En la actualidad, el cultivo de girasol se está desplazando dentro de la misma región debido al daño provocado por la paloma Zenaida auriculata. Ensayos realizados por el INTA Anguil concluyeron que las zonas más afectadas han sido las del oeste y centro de la provincia de $\mathrm{La}$ Pampa, registrando pérdidas máximas que superan los 600 kg.ha-1 (Bernardos \& Farrell, 2012).

El cártamo es un cultivo predominantemente autógamo, con un potencial genético de $90 \%$ de autopolinización aunque, dependiendo de las condiciones ambientales, la polinización cruzada puede llegar hasta el 50\% de tal manera que en ciertas poblaciones de cártamo la homogeneidad se puede perder rápidamente. Las abejas de varios géneros, así como otros insectos, son atraídos por la flor del cártamo para tomar polen y néctar, y son los principales agentes de la polinización cruzada. El viento no es un factor que influye en la polinización cruzada de este cultivo (Muñoz, 1977).

Debido a que en la región semiárida pampeana no existen datos sobre insectos presentes en el cultivo, se consideró importante estudiar tanto las especies que lo atacan, como así también las benéficas, parasitoides y polinizadores. Los objetivos del trabajo fueron: identificar las especies de insectos presentes en el cultivo de cártamo en la región semiárida pampeana y caracterizar su distribución y localización en la planta en los distintos estados fenológicos del cultivo.

\section{Materiales y Métodos}

El ensayo se llevó a cabo en el Campo Experimental de la Facultad de Agronomía de la Universidad Nacional de La Pampa. Dicho predio está situado a $10 \mathrm{~km}$ al norte de la ciudad de Santa Rosa. Latitud: $36^{\circ} 32^{\prime} 55.90^{\prime \prime}$ Sur y Longitud: $64^{\circ} 18^{\prime} 18.00^{\prime \prime}$ Oeste.

El diseño experimental fue realizado en 18 parcelas de $12 \mathrm{~m}^{2}$ cada una ( $\left.2 \times 6 \mathrm{~m}\right)$, las cuales 
recibieron el mismo tratamiento que consistió en una labranza de arada con doble acción, ya que había varias malezas en estado de floración. No se realizó ningún tipo de aplicación de insecticida, ni de fertilizante. Debido a la baja humedad en el suelo a la siembra del cultivo, se realizó riego complementario con un aspersor.

Se utilizó semilla perteneciente al cultivar CW 88-OL con alto contenido oleico, la cual recibió un tratamiento con curasemillas (Carbendazim $10 \%$ + Tiram $10 \%$ ) a una dosis de $300 \mathrm{~cm}^{3}$ cada $100 \mathrm{~kg}$ de semilla.

La siembra se llevó a cabo el día 20/08/2013, con una sembradora convencional para parcelas de ensayos, utilizando $27 \mathrm{~kg}$ de semilla por hectárea. La emergencia se registró el día 12/09/2013, con una densidad obtenida de 30 plantas. $\mathrm{m}^{-2}$. Se realizó un control mecánico de malezas el día $8 / 11 / 2013$, que coincidió con la plena ramificación del cultivo.

El muestreo de insectos se realizó semanalmente, debiendo desplazarse la observación en algunas oportunidades por condiciones de mal tiempo u operativas. Se observaron 10 plantas en cada parcela tomadas al azar desde la emergencia del cultivo hasta madurez fisiológica. En dichas observaciones se analizó la totalidad de la parte aérea de cada planta, realizando la identificación y recuento de los insectos presentes tanto benéficos como perjudiciales. Se determinó, para cada especie encontrada, el número de individuos en 10 plantas. Particularmente para los áfidos, se registró el número de plantas afectadas, el número promedio de pulgones por planta y el número promedio de pulgones por planta infestada (promedio de pulgones con respecto al número de plantas atacadas). Para el análisis de estas tres últimas variables específicas, estadística descriptiva, se utilizó el software Infostat.

El criterio adoptado para establecer los estados

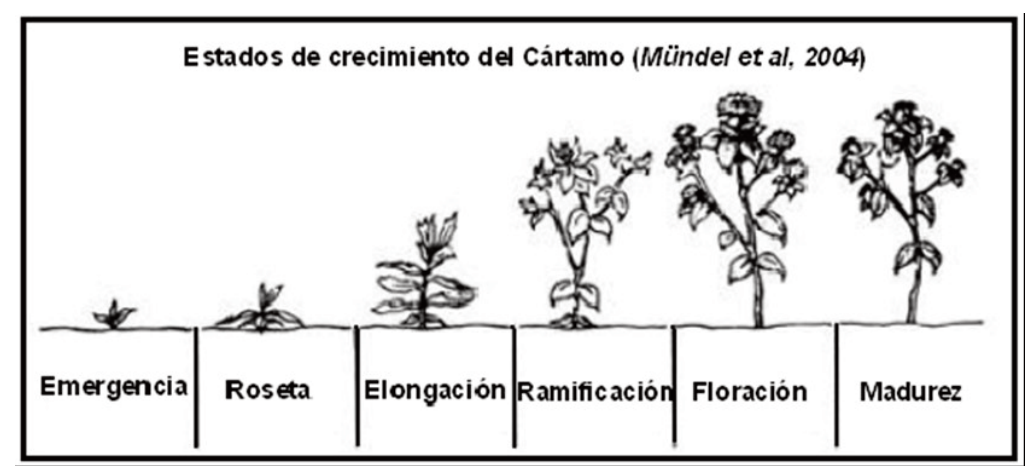

Figura 1. Escala fenológica del cártamo (tomado de Rivas y Matarazzo, 2009). tarazzo, 2009). fenológicos del cultivo de cártamo fue seguir la escala fenológica establecida por Mündel (2004) para los distintos estados de crecimiento de ésta asterácea (Figura 1). Los datos de temperaturas fueron proporcionados por la cátedra de Agrometeorología de la Facultad de Agronomía UNLPam. Tabla 1.

\section{Identificación}

La identificación de las especies de insectos se realizó mediante la utilización de bibliografía específica y claves de reconocimiento: Wharton (1997), Stehr (1991 y 1987), Borror et al (1989), Gauld (1980), De Santis (1967) y Dughetti y La Rosa (2011).

Tabla 1. Datos de temperatura de los meses durante el ensayo. Table 1. Temperature data of the months during the trial.

\begin{tabular}{|c|c|c|c|c|c|}
\hline Año 2013 & Agosto & Setiembre & Octubre & Noviembre & Diciembre \\
\hline $\begin{array}{l}\text { Temperatura } \\
\text { Media }\left({ }^{\circ} \mathrm{C}\right)\end{array}$ & 10,0 & 11,2 & 16,7 & 20,4 & 25,5 \\
\hline $\begin{array}{l}\text { Temperatura } \\
\text { Max. Media }\left({ }^{\circ} \mathrm{C}\right)\end{array}$ & 18,6 & 18,0 & 23,7 & 28,2 & 34,2 \\
\hline $\begin{array}{l}\text { Temperatura } \\
\text { Min. Media }\left({ }^{\circ} \mathrm{C}\right)\end{array}$ & 1,5 & 4,4 & 9,8 & 12,7 & 16,8 \\
\hline
\end{tabular}




\section{Resultados y Discusión \\ Identificación de insectos en el cultivo del Cár- tamo}

A lo largo de todo el periodo de muestreo en el cultivo, se encontraron los siguientes insectos detallados en la tabla 2.

Tabla 2. Listado de las especies de insectos relevados en el cultivo especificando nombre científico, nombre vulgar, ubicación taxonómica y rol ecológico.

Table 2. List of insect species surveyed in the cultivation specifying scientific name, common name, taxonomic status and ecological role.

\begin{tabular}{|c|c|c|c|}
\hline $\begin{array}{l}\text { Rol } \\
\text { Ecológico }\end{array}$ & Nombre científico & Nombre vulgar & $\begin{array}{l}\text { Orden y } \\
\text { Familia }\end{array}$ \\
\hline \multirow{16}{*}{ 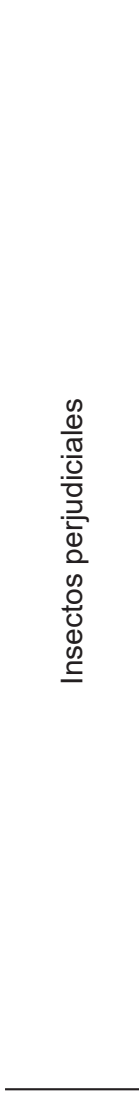 } & Uroleucon jaceae (L.) & Pulgón negro del cártamo & Hemíptera: \\
\hline & $\begin{array}{l}\text { Capitophorus elaeagni (del } \\
\text { Guercio) }\end{array}$ & Pulgón verde del alcaucil & Aphididae \\
\hline & $\begin{array}{l}\text { Athaumasthus haematicus } \\
\text { (Stål) }\end{array}$ & $\begin{array}{l}\text { Chinche roja sanguino- } \\
\text { lenta o chinche del poroto }\end{array}$ & $\begin{array}{l}\text { Hemíptera: Co- } \\
\text { reidae }\end{array}$ \\
\hline & Dichelops furcatus (F.) & Chinche de los cuernos & \multirow{4}{*}{$\begin{array}{l}\text { Hemíptera: Pen- } \\
\text { tatomidae }\end{array}$} \\
\hline & Nezara viridula (L.) & Chinche verde & \\
\hline & Edessa meditabunda (F.) & Alquiche chico & \\
\hline & Piezodorus guildinii (W.) & $\begin{array}{l}\text { Chinche verde pequeña o } \\
\text { Chinche de la soja }\end{array}$ & \\
\hline & Nysius simulans Stål. & Chinche diminuta & \multirow{3}{*}{$\begin{array}{l}\text { Hemíptera: } \\
\text { Lygaeidae } \\
\text { Hemíptera: } \\
\text { Cicadellidae } \\
\text { Thysanoptera: } \\
\text { Thripidae }\end{array}$} \\
\hline & & Chicharritas & \\
\hline & Frankliniella occidentalis P. & $\begin{array}{l}\text { Trips californiano de las } \\
\text { flores }\end{array}$ & \\
\hline & Helicoverpa zea B. & Desgranadora del maiz & \multirow{3}{*}{$\begin{array}{l}\text { Lepidóptera: } \\
\text { Noctuidae }\end{array}$} \\
\hline & Rachiplusia nu (G.) & Oruga medidora & \\
\hline & $\begin{array}{l}\text { Spodoptera frugiperda (J. E. } \\
\text { Smith) }\end{array}$ & Oruga militar tardía & \\
\hline & $\begin{array}{l}\text { Chauliognathus scriptus } \\
\text { (Germ.) }\end{array}$ & Escarabajo escrito & \multirow{3}{*}{$\begin{array}{l}\text { Coleóptera: } \\
\text { Cantaridae } \\
\text { Coleóptera: } \\
\text { Curculionidae } \\
\text { Coleóptera: } \\
\text { Meloidae }\end{array}$} \\
\hline & Pantomorus auripes $\mathrm{H}$. & Gorgojo de la alfalfa & \\
\hline & Epicauta adspersa K. & Bicho moro & \\
\hline \multirow{8}{*}{ 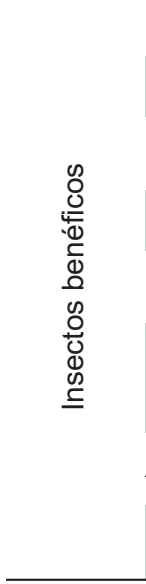 } & Hippodamia convergens G. & Vaquita convergente & \multirow{4}{*}{$\begin{array}{l}\text { Coleóptera: } \\
\text { Coccinellidae }\end{array}$} \\
\hline & Eriopis connexa G. & Vaquita manchada & \\
\hline & Cycloneda sanguinea & $\begin{array}{l}\text { Vaquita roja o de San } \\
\text { José }\end{array}$ & \\
\hline & Harmonia axyridis (P.) & Vaquita & \\
\hline & Nabis sp. & Chinche predadora & $\begin{array}{l}\text { Hemíptera: } \\
\text { Nabidae }\end{array}$ \\
\hline & Ophion sp. & Avispa parasitoide & $\begin{array}{l}\text { Hymenóptera: } \\
\text { Familia? } \\
\text { Ichneumonidae }\end{array}$ \\
\hline & Apis melifera L. & Abejas & $\begin{array}{l}\text { Hymenóptera: } \\
\text { Apidae }\end{array}$ \\
\hline & & Arañas & $\begin{array}{l}\text { Araneae: } \\
\text { Salticidae }\end{array}$ \\
\hline
\end{tabular}

Distribución temporal de áfidos, hemípteros y coccinélidos en relación al estado fenológico de la planta.

\section{Insectos perjudiciales}

Los pulgones se encontraron en mayor proporción con respecto a las otras especies que atacaron al cultivo, luego le siguieron las chinches, los trips y orugas defoliadoras en orden decreciente. Las especies principales encontradas fueron: Uroleucon jaceae (L.) "pulgón negro del cártamo", mencionada a nivel mundial para este cultivo y Capitophorus eleagni (del Guercio) "pulgón verde del alcaucil", atacando a otras especies como el alcaucil (Cynaras colymus). Estos resultados coinciden con Dughetti y Zarate (2011), quienes encontraron que las plagas principales de este cultivo en el sur de la provincia de Buenos Aires son estos áfidos, atacando principalmente en el momento de inicio de floración y afectando significativamente el tercio superior de la planta (hojas, brotes tiernos y capítulos).

\section{Áfidos}

La distribución de los pulgones fue cambiando de acuerdo con el estado fenológico del cultivo (Figura 1). Las colonias se establecieron preferentemente en el tercio superior de la planta en la cara abaxial en las hojas, brotes, tallos tiernos, pimpollos y 


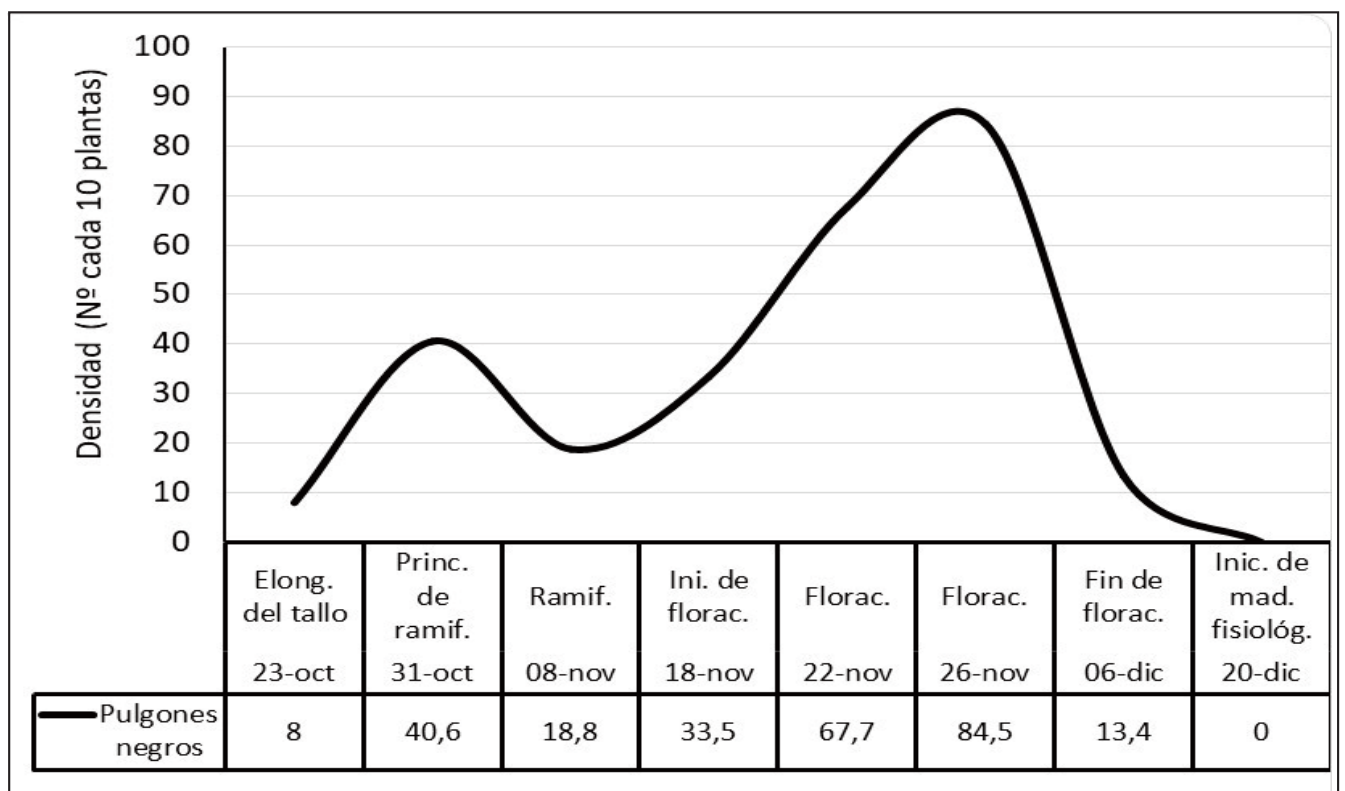

Figura 2. Número de pulgones negros (Uroleucon jaceae (L.)) cada 10 plantas muestreadas. (Facultad de Agronomía, Santa Rosa, La Pampa, temporada 2013-2014). Referencias: Elong. del tallo: Elongación del tallo. Princ. de ramif.: Principio de ramificación. Ramif.: Ramificación. Inic. de florac.: Inicio de floración. Florac.: Floración. Fin de florac.: Fin de floración. Inic. de mad. fisiolog.: Inicio de madurez fisiológica.

Figure 2. Number of black aphids (Uroleucon jaceae (L.)) every 10 plants sampled. (Faculty of Agronomy, Santa Rosa, La Pampa, season 2013-2014). References: Elong. of the stem: Elongation of the stem. Princ. Branch: Branch principle. Ramif: Branch. Start of flowering: Beginning of flowering. Flowering: Flowering. End of flowering: End of flowering. Start from mad. physiology: Beginning of physiological maturity.

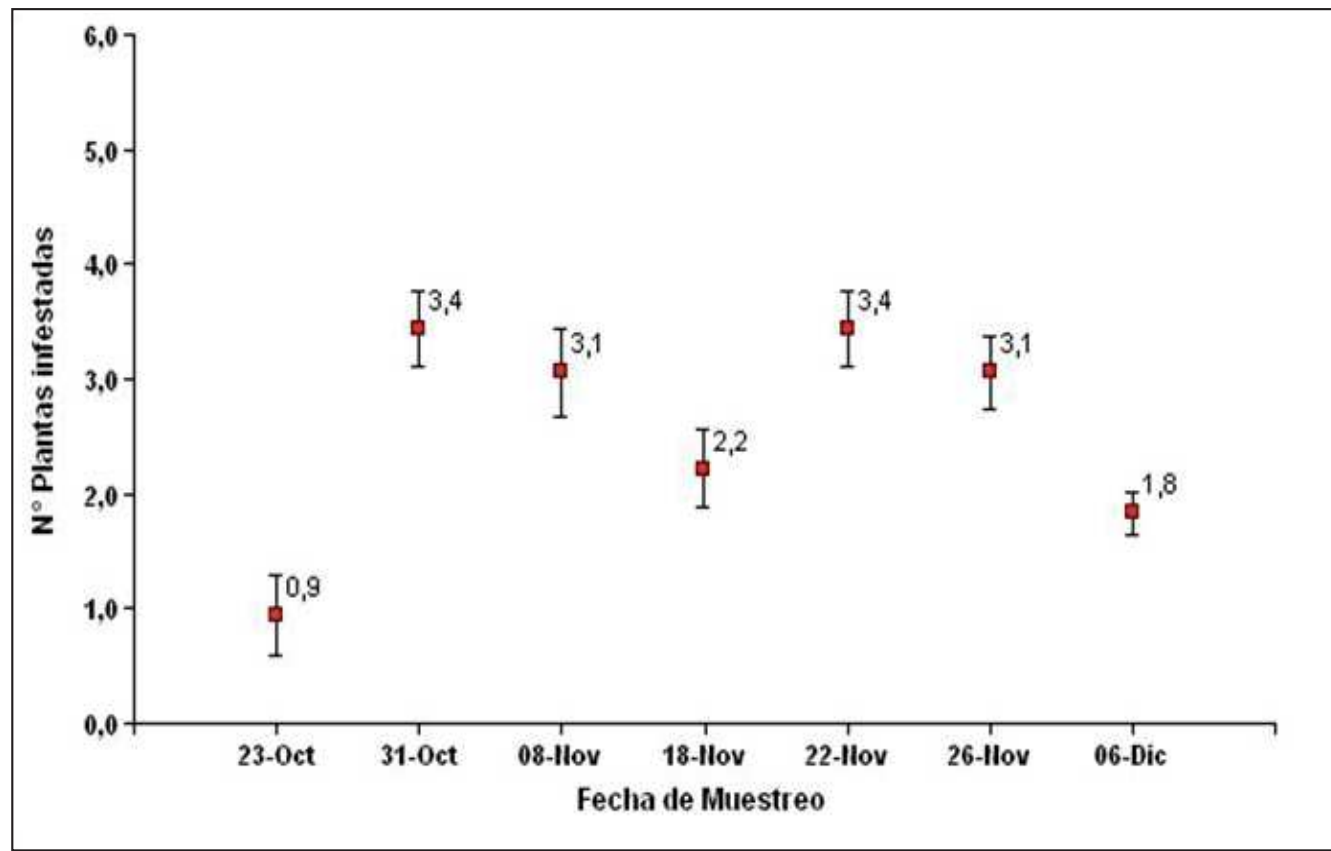

Figura 3. Número promedio de plantas infestadas por Uroleucon jaceae en las distintas fechas de muestreo (Facultad de Agronomía, Santa Rosa, La Pampa temporada 2013-2014).

Figure 3. Average number of infested plants by Uroleucon jaceae at different sampling dates (Faculty of Agronomy, Santa Rosa La Pampa, season 2013-2014). 


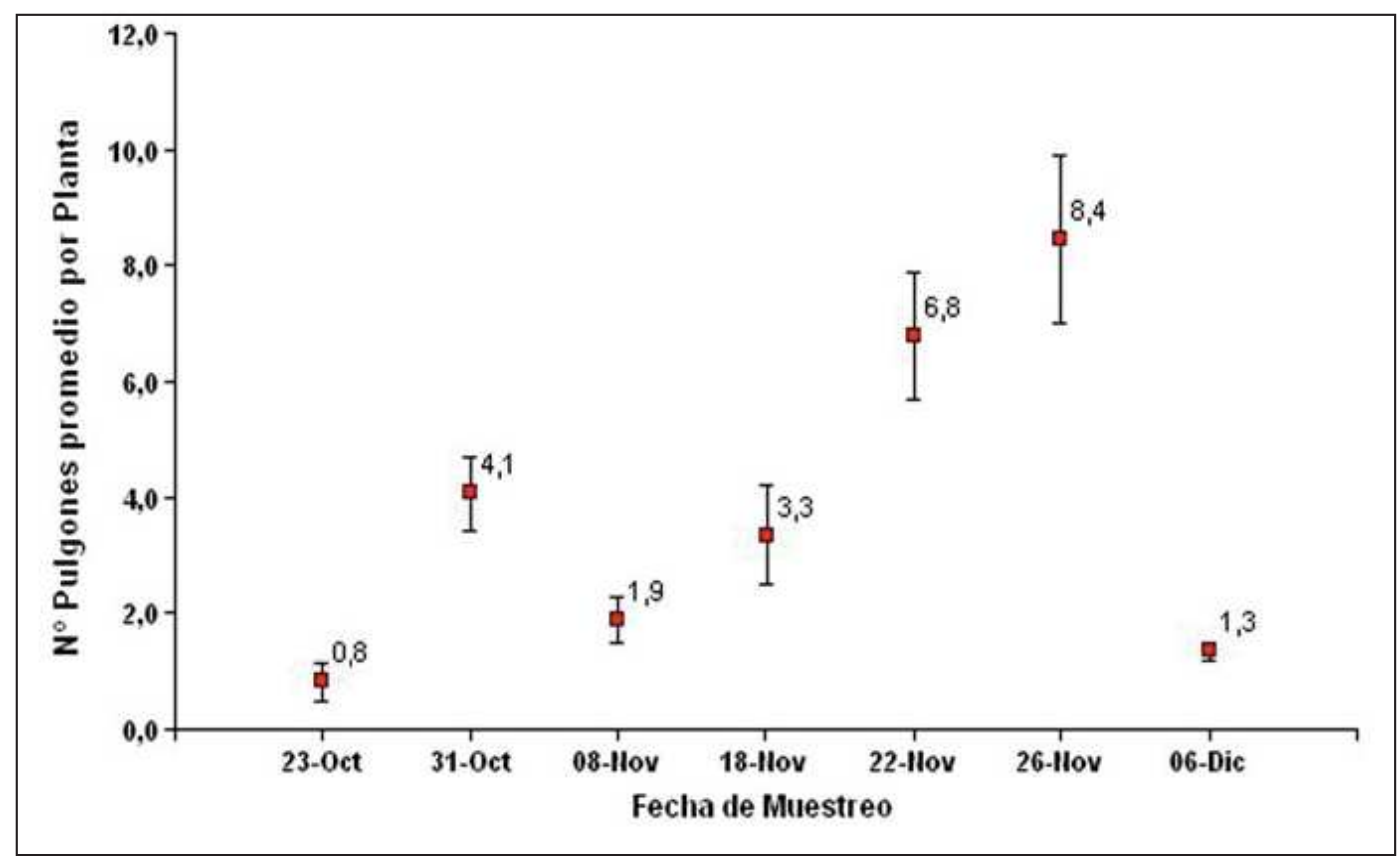

Figura 4. Número promedio de pulgones por planta en las distintas fechas de muestreo. (Facultad de Agronomía, Santa Rosa, La Pampa, temporada 2013-2014).

Figure 4. Average number of aphids per plant in the different sampling dates. (Faculty of Agronomy, Santa Rosa, La Pampa, season 2013-2014).

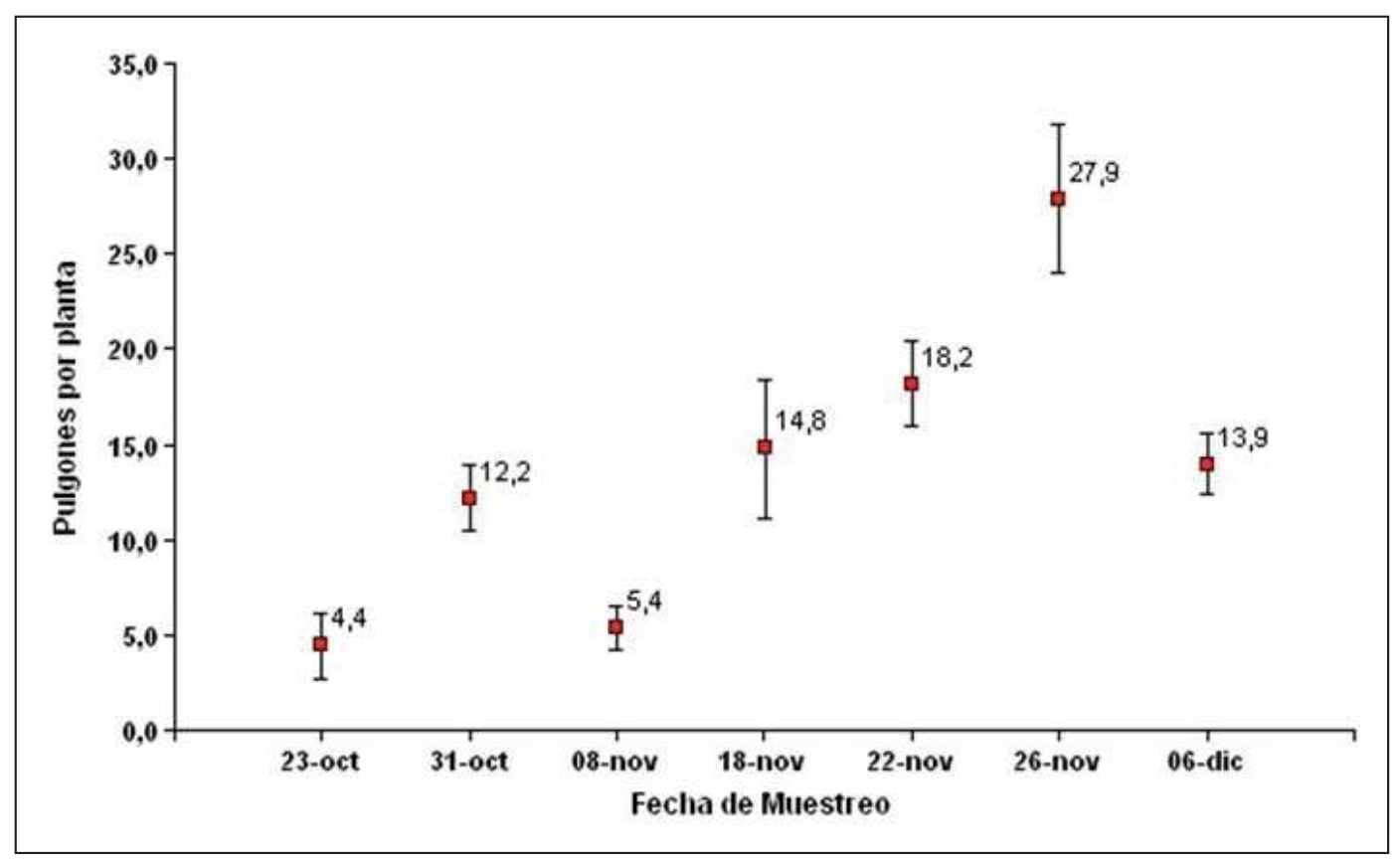

Figura 5. Número promedio de pulgones en plantas atacadas en las distintas fechas de muestreo. (Facultad de Agronomía, Santa Rosa, La Pampa, temporada 2013-2014).

Figure 5. Average number of aphids in plants attacked in the different sampling dates. (Faculty of Agronomy, Santa Rosa, La Pampa, season 2013-2014). 


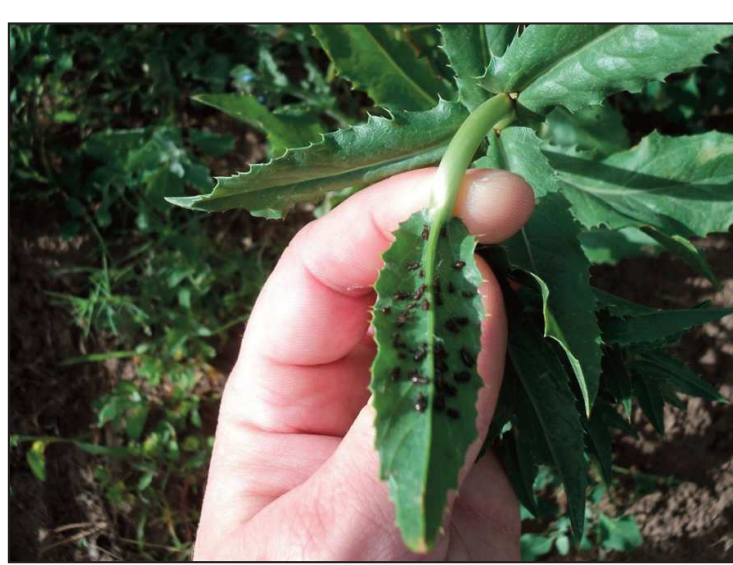

Figura 6. Colonia de Uroleucon jaceae en la cara abaxial de la hoja de cártamo.

Figure 6. Colony of Uroleucon jaceae on the abaxial side of the safflower leaf.

flores, observándose las colonias negras (Figura 6). Dentro del complejo de especies de pulgones presentes, Uroleucon jaceae (L.) se observó con mayor número de colonias que Capitophorus elaeagni (del Guercio). A continuación se presentan las observaciones sobre la primera especie.
La presencia de pulgones fue detectada a partir del 23/10/13 coincidiendo con la elongación del tallo del cultivo. El pico máximo de pulgones se determinó en floración, con 84.5 pulgones cada 10 plantas. Luego, en los siguientes muestreos disminuyó marcadamente hasta la última donde no hubo registros (inicio de madurez fisiológica) (Figura 2).

La Figura 3 indica el número promedio de plantas infestadas de las 10 muestreadas. Como se puede observar, en la primera fecha de muestreo hubo una media de 0.94 plantas infestadas. El mayor número promedio de plantas infestadas fue de 3.44 teniendo dos picos no definidos marcadamente, uno a fines de octubre (principios de ramificación) y otro en noviembre (floración). Es de importancia destacar que el número de plantas infestadas no superó el $40 \%$ durante el ciclo del cultivo.

La Figura 4 muestra el número promedio de pulgones por planta, encontrándose 0.8 pulgones por planta al inicio de la captura (23/10/2013). La población creció hasta el 26/11/2013 (estado de floración) llegando a un máximo de 8,4 pulgones promedio por planta. Luego hacia fin de

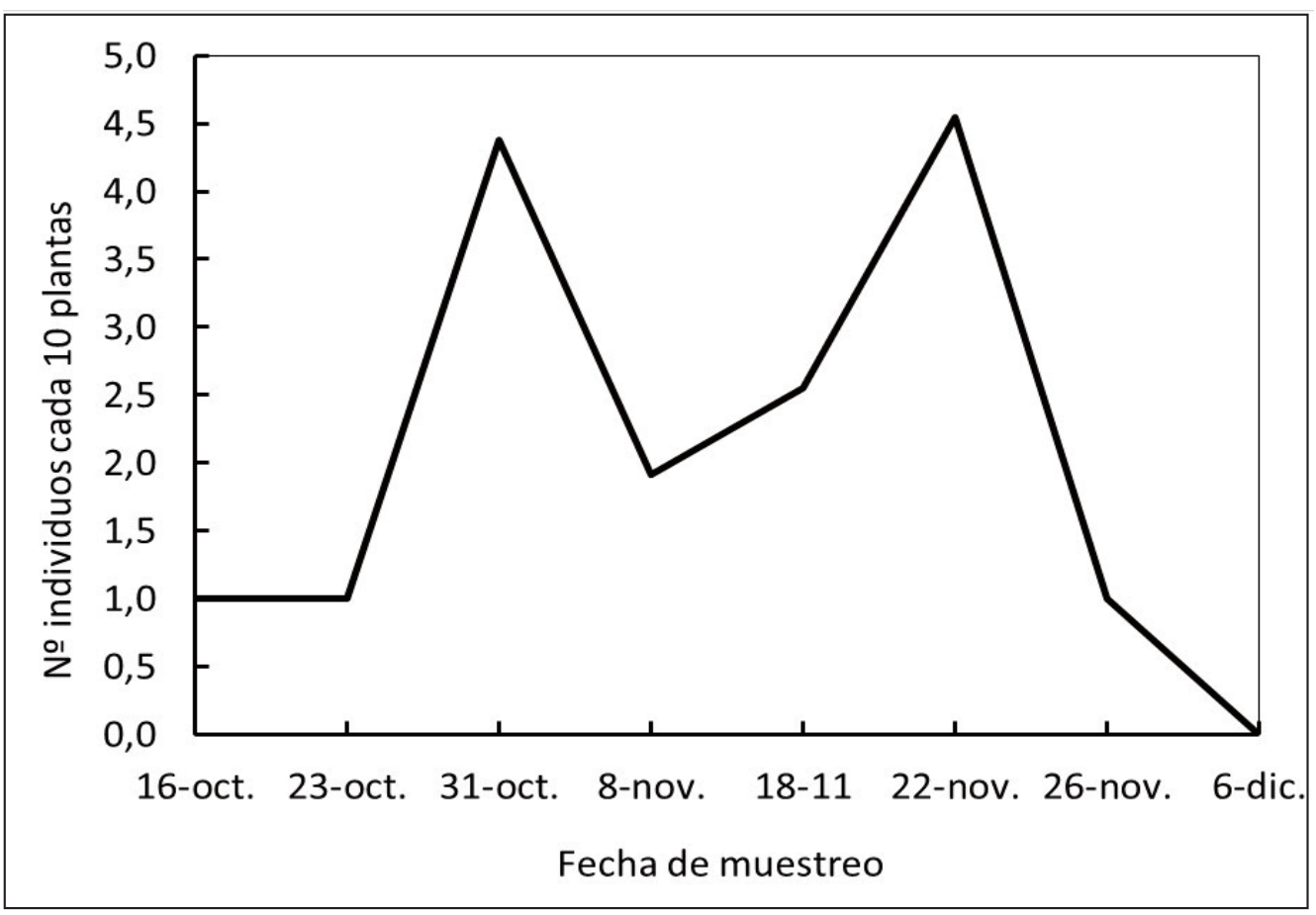

Figura 7. Número de individuos de chinche roja cada 10 plantas muestreadas en las distintas fechas de muestreo (Facultad de Agronomía, Santa Rosa La Pampa, temporada 2013-2014).

Figure 7. Number of individuals of red bugs every 10 plants sampled in the different sampling dates (Faculty of Agronomy, Santa Rosa La Pampa, season 2013-2014). 


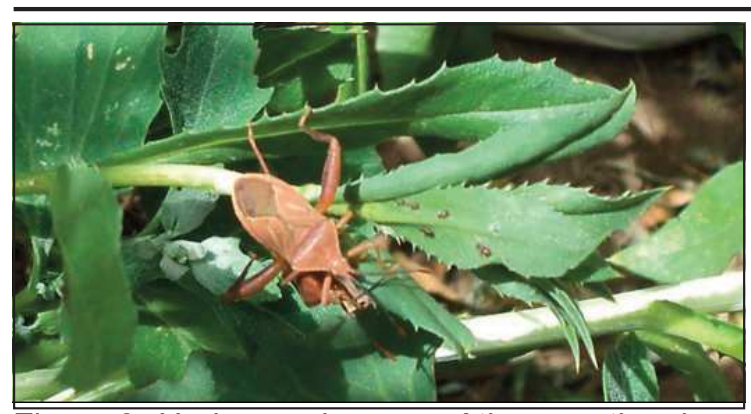

Figura 8. Uroleucon jaceae y Athaumasthus haematicus.

Figure 8. Uroleucon jaceae and Athaumasthus haematicus

floración (06/12/2013) se observaron 1,3 pulgones promedio por planta.

Si tenemos en cuenta solamente las plantas infestadas, el número de pulgones promedio por planta infestada presentó una tendencia lineal creciente desde la elongación del tallo (23/10/2013), habiendo presentado 4,4 pulgones promedio por planta infestada hasta floración (26/11/2013), donde se encontraron 27,9 pulgones promedio por planta infestada (Figura 5). El

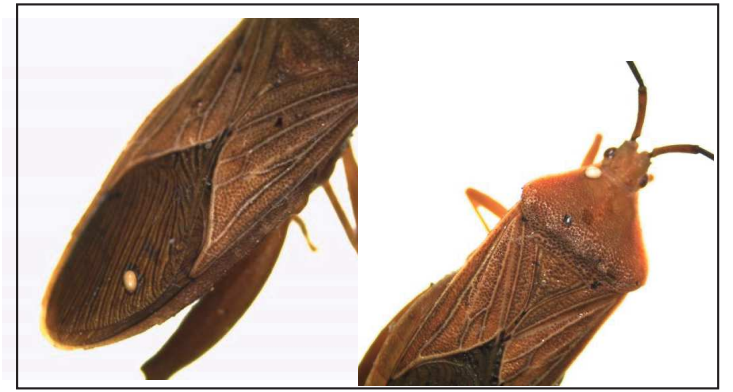

Figura 9. Chinche roja con huevos de dípteros parasitoides.

Figure 9. Red bug with eggs of dipteran parasitoids.

8/11/2013 se observa una disminución en el número promedio de pulgones luego de la ocurrencia de lluvias el 6/11/2013 (17,2 mm). Hacia fin de floración disminuye considerablemente hasta 13,9 pulgones promedio por planta. Hacia el final del ciclo, el cultivo sufrió estrés hídrico por falta de lluvias y altas temperaturas.

\section{Athaumasthus haematicus "chinche roja"}

La presencia de la chinche roja se determinó

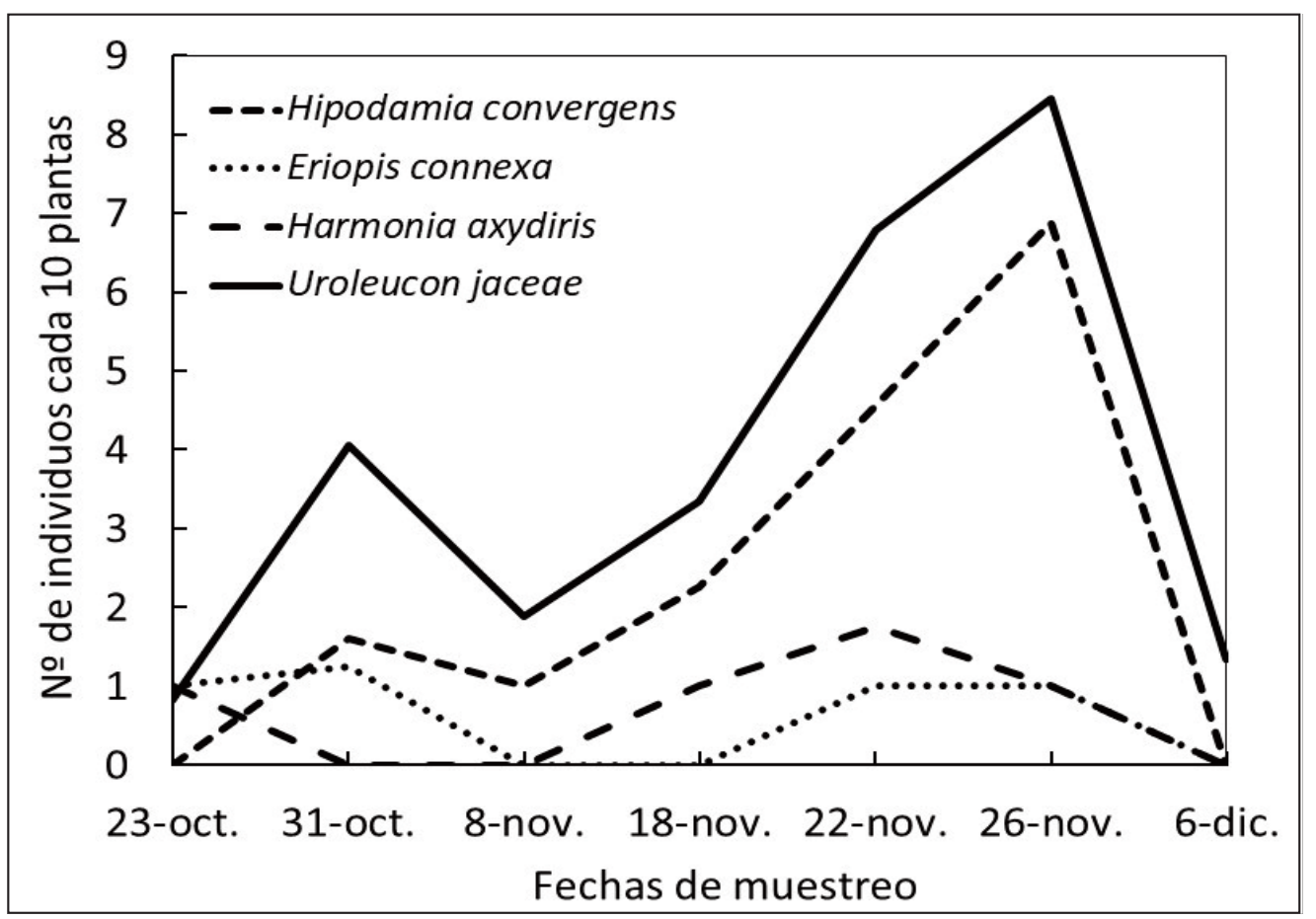

Figura 10. Número de individuos de Hippodamia convergens, Eriopis conexa, Harmonia axyridis y Uroleucon jaceae de en las distintas fechas de muestreo (Facultad de Agronomía, Santa Rosa, La Pampa, temporada 2013-2014).

Figure 10. Number of individuals of Hippodamia convergens, Eriopis conexa, Harmonia axyridis and Uroleucon jaceae from the different sampling dates (Faculty of Agronomy, Santa Rosa, La Pampa, season 2013-2014). 


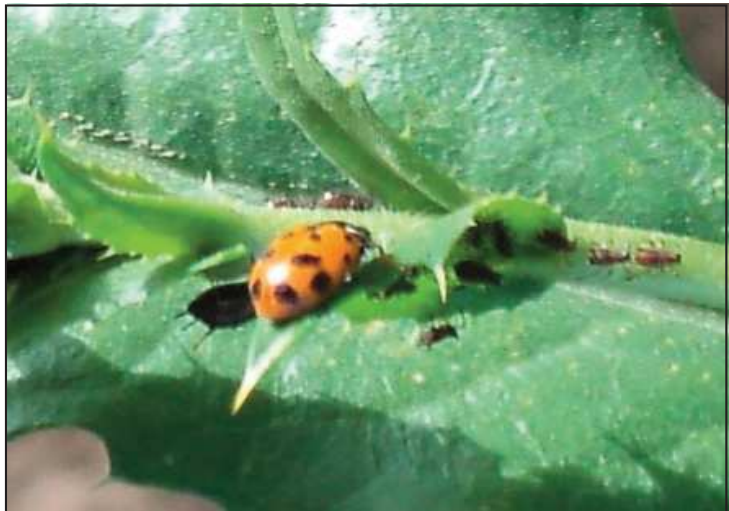

Figura 11. Colonia de Uroleucon jaceae atacada por Hippodamia convergens.

Figure 11. Colony Uroleucon jaceae attacked by Hippodamia convergens.

antes de los pulgones, siendo ésta sobre principio de elongación del tallo (16/10/2013), con un adulto cada 10 plantas. Durante el ciclo del cultivo hubo dos picos bien definidos en el número de individuos, el primero fue en principios de ramificación $(31 / 10 / 2013)$, representada por 4,4 chinches cada 10 plantas y el segundo pico que fue máximo para todo el ciclo, se manifestó el 26/10/2013 (floración) encontrando 4,5 chinches cada 10 plantas (Figura 8).

\section{Insectos benéficos}

Las especies benéficas identificadas de mayor importancia fueron los coccinélidos; con respecto a las restantes se encontraron: abejas; chinches predadoras; avispas parasitoides y arañas.

\section{Coccinélidos}

Dentro de los coccinélidos o vaquitas se destacó, por su densidad poblacional, Hippodamia convergens, luego siguieron Harmonia axyridis y Eriopis connexa en orden decreciente. Estas especies también son citadas, entre otras especies de predadores, para el sur de la provincias de Buenos Aires (Dughetti y Zarate 2011),

El número de individuos de Hippodamia convergens a lo largo del ciclo del cultivo tuvo un comportamiento similar al de los pulgones negros. Desde el comienzo del cultivo, la población no sufrió gran variación manteniéndose relativamente estable con respecto al número de pulgones, llegando hasta su pico máximo en floración $(26 / 11 / 13)$, que también coincide con el pico máximo de número de pulgones cada 10 plantas. Harmonia axyridis, presentó un número de individuos poblacional menor que Hippoda-

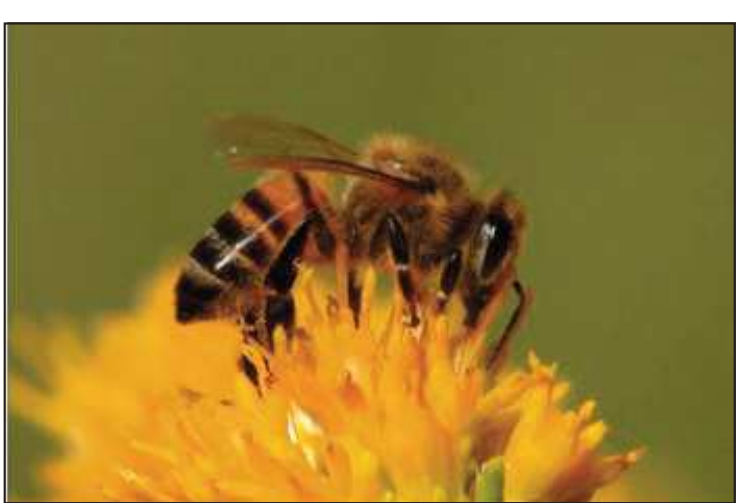

Figura 12. Apis melífera L. en capítulo de Cártamo.

Figure 12. Apis melifera L. in flower heads of Safflower

mia sp., con un pico máximo 4 días antes del máximo correspondiente a los pulgones. Eriopis sp., presentó la menor densidad dentro del complejo de coccinélidos con un número de individuos máximo de 1,2 cada 10 plantas en el ciclo del cultivo.

\section{Apis melífera $\mathbf{L}$.}

En cuanto a este insecto polinizador, se observó su presencia hacia fin de ciclo, específicamente en los dos últimos muestreos realizados hacia fin de floración-inicio de madurez fisiológica (6/12/2013 y 20/12/2013 respectivamente).

\section{CONCLUSIÓN}

De acuerdo al estudio realizado en la región semiárida pampeana, se identificaron: dieciséis especies perjudiciales entre las cuales encontramos 9 pertenecientes al orden Hemiptera (pulgones, chinches y chicharritas), 1 correspondiente al orden Thysanoptera (trips), 3 a Lepidoptera (orugas defoliadoras) y 3 a Coleoptera. En cuanto a las benéficas se encontraron seis especies de insectos, entre las cuales 3 correspondieron al orden Coleóptera (vaquitas predadoras), 1 correspondiente a Hemiptera (chinches predadoras) y 2 a Hymenoptera (avispas parasitoides y abejas polinizadoras), y varias especies de la familia Araneae (predadores).

Se pudieron identificar dos especies de pulgones: Uroleucon jaceae (L.) y Capitophorus elaeagni (del Guercio), con predominio de la primera. Con respecto al pulgón negro del cártamo, el mayor ataque se registró en floración, concentrándose preferentemente en la parte superior de la planta, tallos, brotes tiernos y capítulos. A partir del 20 de diciembre, en madurez fisiológica, no 
se encontraron individuos de pulgones.

Se registraron seis especies más de Hemípteros: Athaumasthus haematicus (Stål), Dichelops furcatus (F.), Nezara viridula (L.), Nysius simulans Stål, Piezodorus guildinii (W.), Edessa meditabunda (F.) y chicharritas. La más importante en cuanto a densidad poblacional fue Athaumasthus haematicus (Stål) "chinche roja sanguinolenta" atacando el cultivo antes que los pulgones, desde principio de elongación del tallo. También se observó que la población tuvo dos picos, uno en principio de ramificación y el segundo, su máximo, en floración.

El complejo de coccinélidos estuvo integrado por Hippodamia convergens, Harmonia axyridis y Eriopis connexa en orden decreciente de densidad. Se observaron predando tanto pulgones adultos como ninfas y huevos. El pico de máxima densidad de Hippodamia convergens coincidió con el pico máximo del pulgón negro, mientras que para Harmonia axyridis su máxima población se manifestó antes del mismo.

Con respecto al orden Hymenóptera, se registraron las especies Ophion sp. Ichneumónido parasitoide) y Apis melifera L. La especie parasitoide se observó por primera vez durante la ramificación y en estado adulto. Apis melífera L. se observó desde floración hasta inicio de madurez fisiológica.

\section{AgradeCIMIENTOS}

Al Sr. Hugo Kaus, por su predisposición y colaboración en la realización de tareas a campo durante todo el ciclo del cultivo. A la familia Fava por donar la semilla para llevar a cabo el trabajo, además de permitir hacer relevamientos de artrópodos en sus lotes con destino comercial. A la Facultad de Agronomía de la Universidad Nacional de La Pampa que nos facilitó el espacio físico, herramientas y materiales para llevar a cabo los ensayos $\mathrm{y}$ las determinaciones posteriores

\section{Bibliografía}

Bernardos J. \& M. Farrell. 2012. Evaluación de daño por paloma torcaza (Zenaida auriculata) en girasol y pérdida de cosecha en la Provincia de La Pampa campaña 2011-2012. INTA EEAAnguil, "Ing. Agr.Guillermo Covas". 21 p.

Borror D.J., C.A. Triplehorn \& N.F. Johnson. 1989. An introduction to the study of insects. Sixth Edition. Saunders College Publishing: 875 p.

Carpintero D. y A. Dughetti. 2012. Presencia de Dersagrena subfoveolata (Berg, 1892) (Hemíptera: Coreidae) en el cultivo de cártamo en el sur de la provincia de Buenos Aires. Revista de Investigaciones Agropecuarias (RIA). BioScriba 5(1): 36-42.

De Santis L. 1967. Catálogo de los himenópteros argentinos de la serie Parasítica, incluyendo Bethyloidea. Provincia de Buenos Aires Gobernación, Comisión de Investigación Científica, La Plata. 337p.

Dughetti A.C. \& R. La Rosa. 2011. Estudio de los áfidos (Hemíptera: Aphididae) que atacan al cártamo (Carthamus tinctorius L.) y sus enemigos naturales en el sur de la provincia de Buenos Aires. Boletin Técnico $N^{\circ} 17.26$ p.

Dughetti A.C. \& A.O. Zarate. 2010-2011.Resultados de la actividad con el cultivo de cártamo. Convenio oleaginosa moreno SA EEA INTA Hilario Ascasubi. 28 p.

Emongor V. 2010. Safflower (Carthamus tinctorius L.) the Underutilized and Neglected Crop: A Review. Departament of crop Sciencie and Production, Botswana College of Agriculture, Private Bag 0027, Gaborone, Botswana. Asian j. Plant Sci. 9(6): 299-306.

Frasier I.M. Lang. 2009. Informe de Campaña. Cártamo 2009. Secretaría de Agricultura, Ganadería, Pesca y Alimentación. Instituto Nacional de Tecnología Agropecuaria. Centro Regional La Pampa - San Luis. 7 p.

Gauld I.D. 1980. An analysis of the classification of the Ophion genus-group (Ichneumonidae). System. Entom. 5: 59-82.

Mündel H.H., R.E. Blackshaw, J.R. Byers, H.C. Huang, D.L. Johnson, R. Keon, J. Kubik, R. McKenzie, B. Otto, B. Roth \& K. Stanford. 2004. Safflower Production on the Canadian Prairies. Agriculture and Agri-Food Canada, Lethbridge Research Centre, Lethbridge, Alberta.

Muñoz B.S. 1977. Determinación del porcentaje de polinización natural en cártamo en el Valle del Yaqui, Son. En: Avances de la InvestigaciónCIANO. O-I 1976-1977 (M.F. Pacheco ed.). p. 1.

Rivas J. \& R. Matarazzo. 2009. Producción de Cártamo, Consideraciones Generales. Boletín de divulgación $N^{\circ} 20$. EEA INTA Hilario Ascasubi. 19 p.

Stehr F.W. 1987. Order Lepidoptera: 288-596. En: Immature insects (F.W. Stehr ed.), Volume 1. Dubuque, Kendall/Hunt Publishing Company, $754 p$.

Stehr F.W. 1991. Immature Insects. Volume 2. Kendall / Hunt Publishing Company. 996 pp.

Wharton R.A., P.M. Marsh \& M.J. Sharkey. 1997. Manual of the new world genera of the family Braconidae (Hymenoptera). The International Society of Hymenopterists. Washington, DC. 439 p. 